Total mensuel des précipitations : $80 \%$ de la normale Fraction d'insolation moyenne : excédentaire

Température moyenne : $>0,9^{\circ} \mathrm{C}$ à la normale

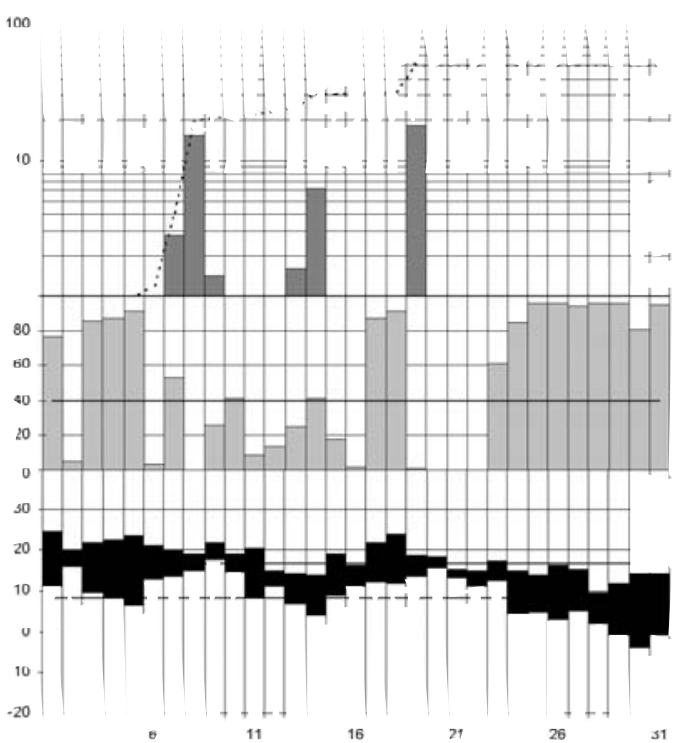

Total mensuel des précipitations : normal Fraction d'insolation moyenne : excédentaire

Température moyenne : $<0,2^{\circ} \mathrm{C}$ à la normale

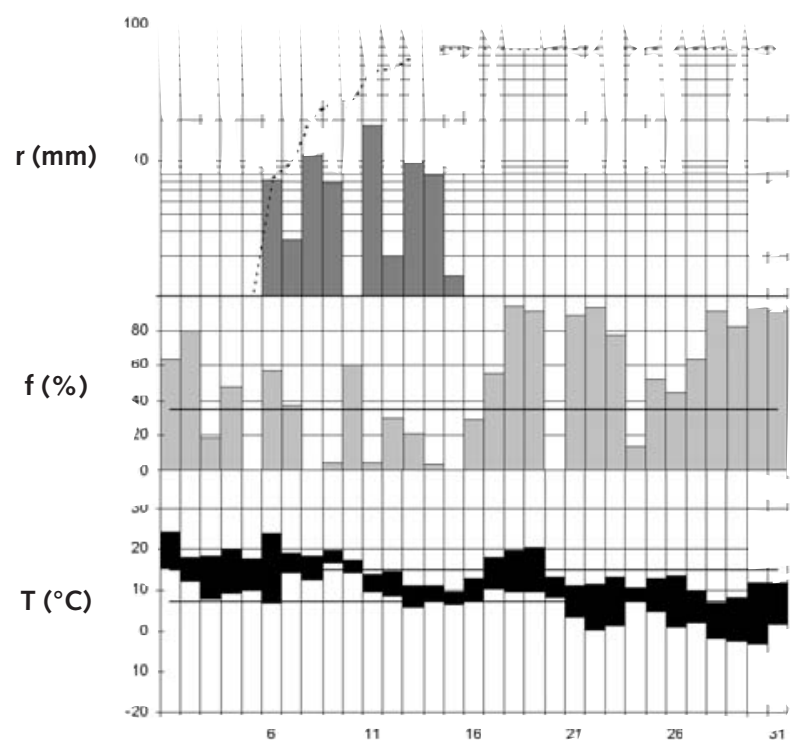

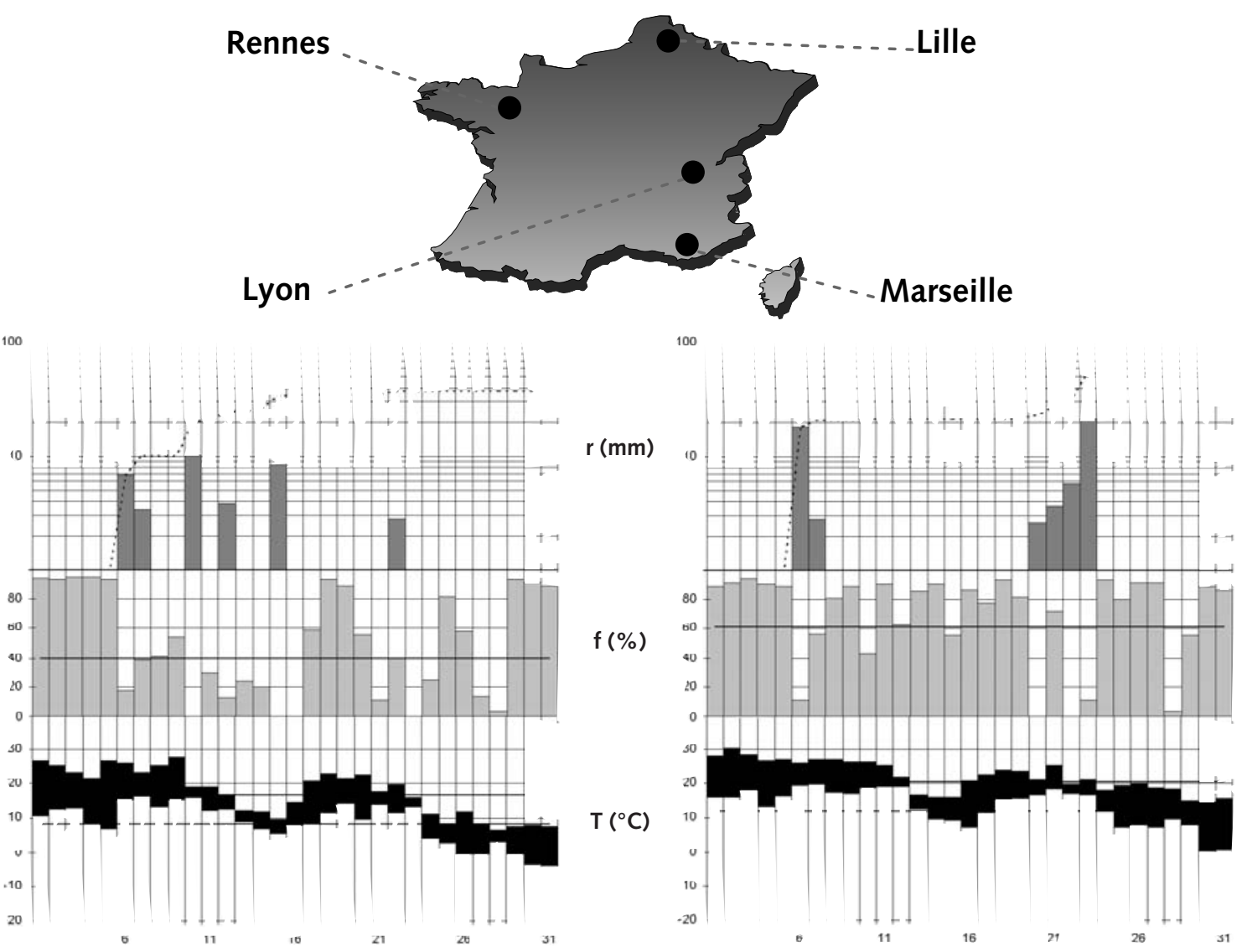

jours

Total mensuel des précipitations : la moitié de la normale Fraction d'insolation moyenne : excédentaire

Température moyenne : $>0,4{ }^{\circ} \mathrm{C}$ à la normale

Total mensuel des précipitations : $70 \%$ de la normale Fraction d'insolation moyenne : légèrement excédentaire

Température moyenne : $>1,7^{\circ} \mathrm{C}$ à la normale

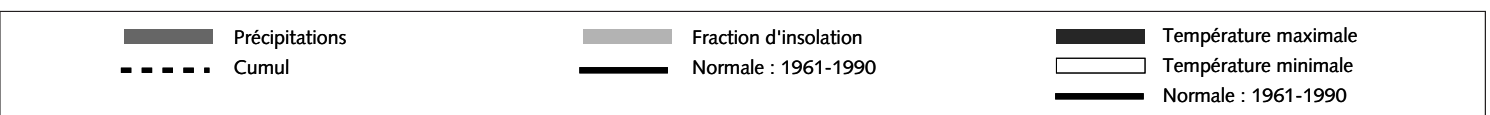


Total mensuel des précipitations : normal Fraction d'insolation moyenne : excédentaire

Température moyenne : normale

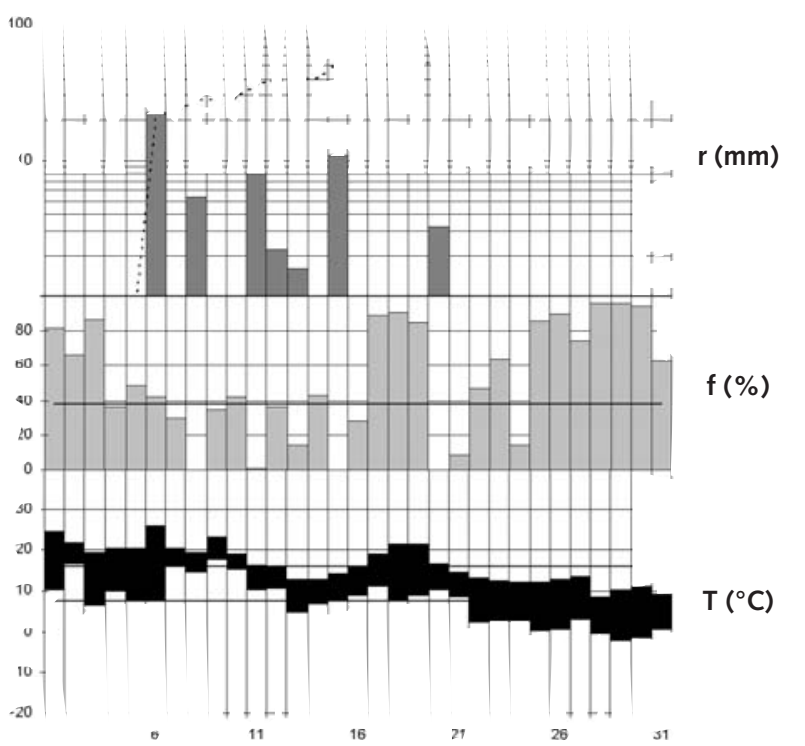

Total mensuel des précipitations : $70 \%$ de la normale Fraction d'insolation moyenne : excédentaire

Température moyenne : $<0,4^{\circ} \mathrm{C}$ à la normale

100

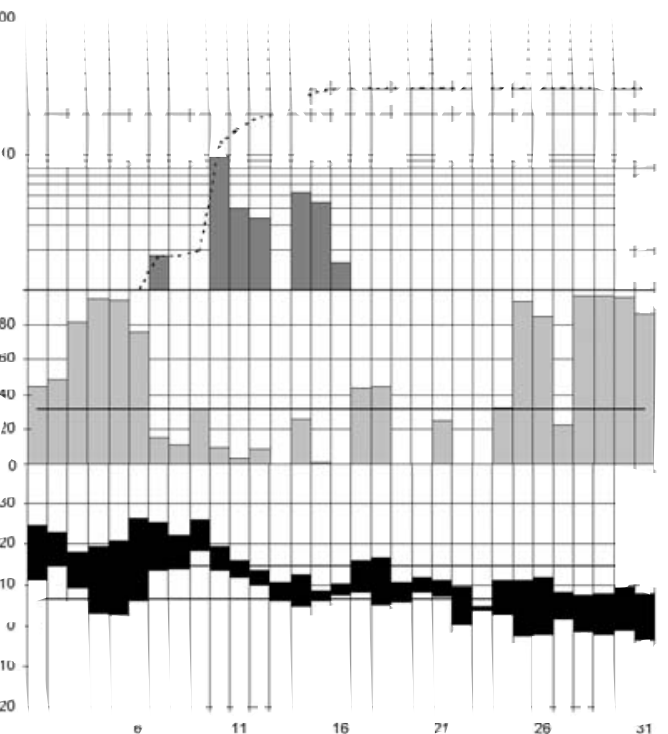

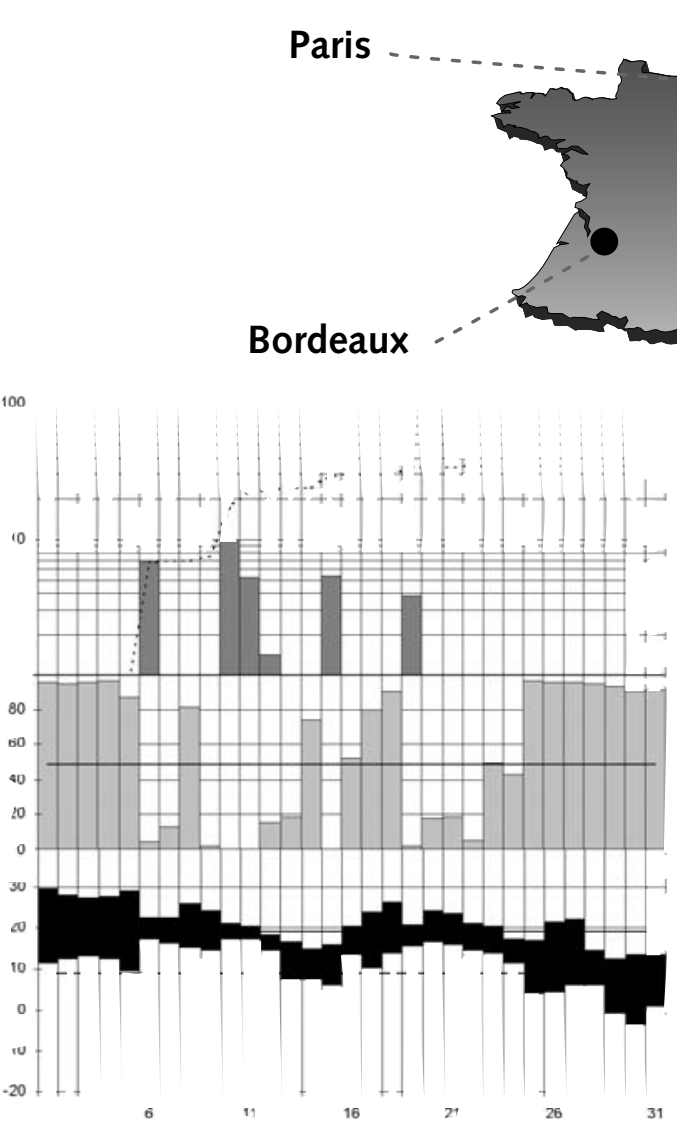
$r(\mathrm{~mm})$

100 $f(\%)$

Total mensuel des précipitations : $40 \%$ de la normale Fraction d'insolation moyenne : excédentaire

Température moyenne : $>2{ }^{\circ} \mathrm{C}$ à la normale

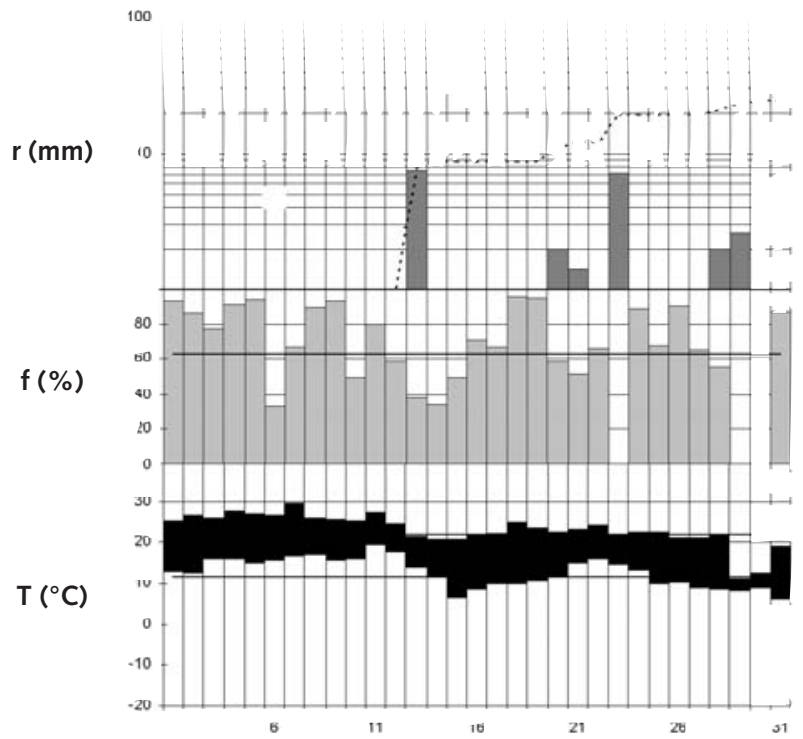

Total mensuel des précipitations : $30 \%$ de la normale Fraction d'insolation moyenne : normale

Température moyenne : $>1,2^{\circ} \mathrm{C}$ à la normale 


\section{octobre 1997}

(Heures UTC sur tout le bulletin)

\section{Périodes thermiques}

\section{Un mois tres contrasté}

La situation météorologique offre des périodes très tranchées. De nombreux records mensuels de température sont battus : en début de mois à cause des températures élevées, puis en fin de mois à cause du froid. La première période chaude se caractérise par des conditions anticycloniques suivies d'un temps perturbé d'ouest très doux. Du 13 au 16, le courant de nord rafraîchit nettement l'atmosphère. Ensuite, la douceur revient grâce au flux de sud présent sur la façade atlantique. À partir du 22, les hautes pressions organisent peu à peu sur le pays un courant d'est froid à partir du nord-est.

Du $1^{\text {er }}$ au 12 : impression estivale

Dans la continuité du mois de septembre, ces douze premiers jours d'octobre sont d'une grande douceur sur l'ensemble du pays, comme le démontrent de nombreux records de chaleur. Par exemple, la moyenne des températures maximales de cette première décade est battue à Cannes avec $+25,4{ }^{\circ} \mathrm{C}$ (précédent record : $+24,3{ }^{\circ} \mathrm{C}$ en 1986) ou encore à Dijon avec $+22,3^{\circ} \mathrm{C}$ (précédent record : $+22,1^{\circ} \mathrm{C}$ en 1947). Toutefois, cette période de chaleur s'organise différemment suivant les régions. Sur le Sud-Ouest et le pourtour méditerranéen, toute la période se situe au-dessus des normales de saison avec un écart moyen de +4 à $+5^{\circ} \mathrm{C}$. Sur toutes les autres régions, on note un retour à la normale les 3 et 4 et un pic de chaleur le 9, avec un écart maximal à la normale de $1^{\prime}$ ordre de $+6{ }^{\circ} \mathrm{C}$ pour l'Ouest, $+7{ }^{\circ} \mathrm{C}$ pour le Nord et le CentreEst et jusqu'à $+9^{\circ} \mathrm{C}$ pour le Nord-Est.

Le $1^{\text {er }}$, les températures maximales sont très élevées sur le pays. Elles s'échelonnent $\mathrm{de}+24^{\circ} \mathrm{C}$ à Lille à $+33^{\circ} \mathrm{C}$ à Perpignan. Un record mensuel est battu en Bretagne à Saint-Nazaire avec $+26,9{ }^{\circ} \mathrm{C}$ (précédent record : $+26,2{ }^{\circ} \mathrm{C}$ en 1986). Le 2 , de nombreux records mensuels tombent dans le Sud-Est. Les stations météorologiques enregistrent au plus chaud de l'après-midi : $+31,8^{\circ} \mathrm{C}$ à Montpellier (précédent record : $+29,8{ }^{\circ} \mathrm{C}$ en 1983$),+29,8{ }^{\circ} \mathrm{C}$ à SaintAuban dans les Alpes-de-Haute-Provence (précédent record : $+27,8^{\circ} \mathrm{C}$ en 1980) ou encore $+25,8^{\circ} \mathrm{C}$ à Millau (précédent record : $+24,6^{\circ} \mathrm{C}$ en 1979 ).

Les 3 et 4, rafraîchissement temporaire sur le Nord et l'Est du pays avec l'orientation des vents de basses couches au nord-est.
Le thermomètre chute et affiche au petit matin parfois seulement $+1{ }^{\circ} \mathrm{C}$ comme à Reims le 3 ou à Nevers le 4 . À l'inverse, pour ces mêmes régions, le 9 est la journée la plus chaude, mais pas forcément la plus ensoleillée. Le temps est perturbé, très venté dans un rapide flux de sud-ouest. Les minimales sont très élevées, avec en moyenne $+17^{\circ} \mathrm{C}$, et les maximales sont le plus souvent comprises entre $+23{ }^{\circ} \mathrm{C}$ et $+26{ }^{\circ} \mathrm{C}$.

Le début de matinée du 10 est encore plus doux que la veille. À cette occasion, plusieurs records de température minimale sur une période de 7 jours sont dépassés comme à Villacoublay : $+15,9^{\circ} \mathrm{C}$ (précédent record : $+15,1^{\circ} \mathrm{C}$ en 1970) ou à Limoges : $+16,0{ }^{\circ} \mathrm{C}$ (précédent record : $+15,5^{\circ} \mathrm{C}$ en 1973 ).

\section{Deux à quatre jours de fraîcheur}

Le rapide flux d'altitude bascule de l'ouest au nord, apportant un rafraîchissement assez brutal. Les températures maximales des 13 et 14 pour l'Ouest, des 13, 14 et 15 pour le Nord et le Sud-Ouest, et des 13, 14,15 et 16 pour le Nord-Est, le CentreEst et les régions méditerranéennes ressemblent aux températures minimales d'il $\mathrm{y}$ a quelques jours à peine.

\section{Grande douceur ensuite}

Grande douceur de la mi-octobre au 21 pour le Nord et le Nord-Est, au 23 pour l'Ouest et le Centre-Est, au 24 pour le SudOuest et au 25 pour le Sud-Est et la Corse. Durant cette période, le courant se rétablit au sud. Les températures en profitent pour grimper. Les nuits ne sont pas froides : il fait le plus souvent entre $+7^{\circ} \mathrm{C}$ et $+15^{\circ} \mathrm{C}$. Les aprèsmidi des 18, 19 et 20 sont les plus doux en général : les maximales s'étagent entre $+20^{\circ} \mathrm{C}$ et $+24^{\circ} \mathrm{C}$ du Nord au Sud du pays, exception faite de Strasbourg où le thermomètre se bloque à une douzaine de degrés sous des brouillards tenaces. Le 18, le thermomètre s'envole sur l'Aquitaine par effet de foehn avec +26 à $+28^{\circ} \mathrm{C}$. La Rochelle bat un record de température maximale élevée sur une période de 7 jours : $+25,9{ }^{\circ} \mathrm{C}$ (précédent record : $+25,0^{\circ} \mathrm{C}$ en 1988). La journée du 21 reste chaude pour la saison sur les régions méridionales alors que, sur le Nord du pays, les températures amorcent une baisse. On enregistre un record de température maximale sur une période de 7 jours à Istres dans les Bouches-du-Rhône avec $+26,2{ }^{\circ} \mathrm{C}$ (précédent record : $+25,0^{\circ} \mathrm{C}$ en 1921 ). 
Une fin de mois bien froide

À partir du 22, de l'air froid et sec s'infiltre petit à petit sur le pays à partir du nord et de l'est. Les 24 et 25 , avec un anticyclone à $1030 \mathrm{hPa}$ sur les îles Britanniques, un courant d'est s'établit en basses couches sur le Nord de la France. Des gelées matinales apparaissent alors et le thermomètre ne dépasse pas $+12{ }^{\circ} \mathrm{C}$ sur la moitié nord du pays. Le 26, l'air froid a gagné les régions méditerranéennes. À partir du 28, le froid s'accentue. Les gelées nocturnes deviennent plus intenses. Le 30 , de très nombreux records mensuels de température minimale basse sont battus dans tout l'Hexagone. En voici quelques-uns : $-6,3{ }^{\circ} \mathrm{C}$ à BâleMulhouse (précédent record : $-6,1{ }^{\circ} \mathrm{C}$ en 1950), $-5,0^{\circ} \mathrm{C}$ à Bourges (précédent record : $-4,6{ }^{\circ} \mathrm{C}$ en 1940 ) ou encore $-3,1{ }^{\circ} \mathrm{C}$ à Carpentras (précédent record : $-1,8{ }^{\circ} \mathrm{C}$ en 1974). À Clermont-Ferrand, c'est le 29 que le record tombe avec $-9,2{ }^{\circ} \mathrm{C}$ (ancien record : $-8,2{ }^{\circ} \mathrm{C}$ en 1949). Quant aux maximales, elles s'échelonnent le plus souvent entre +7 et $+16{ }^{\circ} \mathrm{C}$ du nord au sud. À Saint-ÉtienneBouthéon, à cause du manque d'ensoleillement, l'après-midi du 28 est le plus froid d'un mois d'octobre depuis l'ouverture de la station en 1950 avec $+2,2{ }^{\circ} \mathrm{C}$ (précédent record : $+2,6^{\circ} \mathrm{C}$ en 1950$)$.
Périodes pluviométriques
Dans la continuité du mois de septembre, le début de ce mois d'octobre est sec. Puis, à partir du 6 et jusqu'au 22, huit perturbations traversent le pays dans un flux de secteur ouest ondulant. Le temps sec reprend le dessus pour la fin du mois, excepté pour les régions Sud-Est et Corse.

\section{Séquences pluvieuses}

Du $1^{\text {er }}$ au 5 : temps sec

Les conditions anticycloniques sont bien installées pour ce début de mois. Les perturbations sont rejetées au-delà de nos frontières. On relève juste quelques pluies à peine significatives sur les régions Ouest et Nord-Est.

Les 6 et 7 : bien arrosé sur la région Sud-Est

Les hautes pressions se décalent vers l'Europe centrale, laissant ainsi la place à un régime perturbé de sud-ouest. Des remontées d'air chaud et instable s'installent à l'avant. Au cours de cette courte période, aucune région n'échappe à la pluie. Sur la région Sud-Est, le 6 et la nuit suivante, il pleut parfois fort et avec des orages localement violents sur les versants sud du relief des Cévennes. On recueille $23 \mathrm{~mm}$ en moyenne sur cette région, soit presque la moitié des pluies de ce mois (48 mm).

Quelques valeurs remarquables en 24 heures: le 6, Les-Vans : $194 \mathrm{~mm}$ (dont $68 \mathrm{~mm}$ en une heure), La Palud : $188 \mathrm{~mm}$, La GrandeCombe : $183 \mathrm{~mm}$, Donzère : $150 \mathrm{~mm}$, Villefort : $107 \mathrm{~mm}$, Tarascon (Bouches-duRhône) : 102 mm. Le 7, Saint-Martin-deCrau : 75 mm, La Motte-du-Caire : $56 \mathrm{~mm}$. Sur les autres régions, on recueille respectivement $12 \mathrm{~mm}$ sur les régions Ouest et Nord, 6 à $9 \mathrm{~mm}$ sur les régions Nord-Est, Centre-Est et Sud-Ouest. Sur la Corse, il est tombé en moyenne $2 \mathrm{~mm}$ d'eau. Par contre, des orages localement forts se sont produits, comme à Solenzara, le 6, avec $118 \mathrm{~mm}$ d'eau recueillie. Du 8 au 15 : temps perturbé

La période du 8 au 12 est dominée par un flux d'ouest dans lequel circulent les perturbations océaniques. Puis, du 13 au 15 , un courant plus frais de nord-ouest perturbé s'installe.

C'est la région Nord-Est qui est la plus arrosée avec en moyenne $43 \mathrm{~mm}$ d'eau recueillie. Les 10 et 11, la pluie tombe parfois de manière soutenue (on recueille en moyenne $23 \mathrm{~mm}$ pour ces deux jours). Le 10 , on mesure respectivement $81 \mathrm{~mm}$ à Lepuix et $62 \mathrm{~mm}$ à Masevaux.

Sur les régions Nord et Ouest, il est tombé $36 \mathrm{~mm}$ en moyenne. On retiendra particulièrement pour la région Ouest la journée du 8 qui est la plus arrosée du mois avec $18 \mathrm{~mm}$ en moyenne. On recueille ce jour $72 \mathrm{~mm}$ à Plouray, $70 \mathrm{~mm}$ à Cherbourg et $62 \mathrm{~mm}$ à Landivisiau.

Sur la région Centre-Est et la région SudOuest, la quantité d'eau recueillie atteint $23 \mathrm{~mm}$ pour la période. C'est la journée du 10 qui est la plus arrosée.

À noter que le temps reste pratiquement $\mathrm{sec}$ sur la région Sud-Est (moins de $1 \mathrm{~mm}$ d'eau recueillie sur la période).

Sur la Corse, une dépression située entre la Sardaigne et l'Italie engendre des retours instables les 13 et 14 . Il tombe $19 \mathrm{~mm}$ d'eau en moyenne au cours de ces deux jours, soit la totalité de la période. Les premiers flocons sont arrivés sur la Corse le 14 au-dessus de 1700 mètres d'altitude.

Du 16 au 18 : temps plus calme

Les hautes pressions reprennent temporairement le dessus, puis baissent le 18 .

Du 19 au 23 : retour de la pluie

Une dépression sur le proche Atlantique, associée à une goutte froide, reste quasi stationnaire. Les masses nuageuses qui lui sont associées intéressent principalement les régions situées sur l'Ouest et le Sud du pays. Sur les régions Nord, Nord-Est et CentreEst, l'activité pluvieuse de ces nuages reste faible (1 à $2 \mathrm{~mm}$ en moyenne).

Sur la région Sud-Ouest, on recueille $6 \mathrm{~mm}$ d'eau en moyenne. Sur la région Ouest, la 
hauteur d'eau atteint $19 \mathrm{~mm}$, dont $10 \mathrm{~mm}$ pour la journée du 19 où des averses orageuses assez intenses se produisent. La région Sud-Est recueille $18 \mathrm{~mm}$ de pluie en moyenne au cours de cette période. Ces pluies tombent surtout sous forme d'averses à caractère orageux. Elles atteignent des quantités importantes par endroits, comme le 20 à Collobrières (84 $\mathrm{mm}$ ) et à Menton (42 mm).

Sur la Corse, on recueille $22 \mathrm{~mm}$ en moyenne sur la période, mais ce sont les journées des 21 et 23 qui sont les plus arrosées, principalement sous les orages. On recueille notamment le 21, à Solenzara, $46 \mathrm{~mm}$ de pluie et le 23 à Bastia, $60 \mathrm{~mm}$ de pluie.
Du 24 au 31 : temps sec excepté sur les régions Sud-Est et Corse

Pour les régions Nord, Nord-Est, CentreEst, Ouest et Sud-Ouest, le temps sec et frais s'installe jusqu'à la fin de ce mois.

Du 27 au 30, un temps pluvio-instable sévit en Méditerranée ; il concerne particulièrement la Corse. La quantité de pluie recueillie en moyenne atteint $18 \mathrm{~mm}$ au cours de cette période. Les pluies sont parfois fortes et orageuses, comme le 27 au cap Sagro, où la hauteur d'eau recueillie atteint $65 \mathrm{~mm}$; le 28, il est tombé $50 \mathrm{~mm}$ d'eau à Solenzara.

À noter que ces pluies touchent la région Sud-Est le 28 où l'on recueille en moyenne $5 \mathrm{~mm}$ d'eau. Le temps est resté sec les autres jours de la période.
La température

Les précipitations

L'ensoleillement

Le vent

Les vents sont assez calmes pour le début du mois : on relève au plus quelques rafales jusqu'à $110 \mathrm{~km} / \mathrm{h}$ au mont Aigoual le 6 .

À partir du 7, le flux de sud-ouest devient plus rapide jusqu'au 9. On enregistre des
Le cumul mensuel des précipitations varie de 7,8 mm à Agen (Lot-et-Garonne) à 262,4 $\mathrm{mm}$ à Solenzara (Corse). Il est déficitaire sur la majeure partie de l'Hexagone avec un déficit maximal de $83 \%$ à La Bastide-desJourdans dans le Vaucluse. Seuls le Cotentin, le Nord, le centre de la Bretagne et une partie de la Corse enregistrent des excédents. C'est en Corse que l'on relève le plus fort excédent avec $111 \%$.

Le nombre de jours avec précipitations supérieures ou égales à $1 \mathrm{~mm}$ varie de 2 à Cannes (Alpes-Maritimes), Perpignan et
La durée d'insolation de ce mois d'octobre varie de 127,3 heures à Cherbourg (Manche) à 232,7 heures à Marignane (Bouches-duRhône). Elle est supérieure à 200 heures sur l'extrême Sud-Est. Quelques records de durée
C'est dans le Sud-Ouest que l'on relève les plus forts excédents (supérieurs à $1,5^{\circ} \mathrm{C}$ ).

Lyon-Satolas affiche deux records paradoxaux : le nombre de jours avec gel sous abri : 4 (ancien record : 2 en 1983, 1985 et 1991) et le nombre de jours avec température maximale supérieure ou égale à $25^{\circ} \mathrm{C}$ : 5 (ancien record : 4 en 1990).
Vernet-les-Bains (Pyrénées-Orientales) à 13 à Louargat, Rostrenen (Côtesd'Armor), Pougne-Hérisson (Deux-Sèvres) et Plouray (Morbihan). Ce nombre de jours est généralement déficitaire à l'exception des Pays de la Loire, d'une partie du Cotentin, de la région de CharlevilleMézières, de la région de Chamonix, de la côte varoise et de la Corse. Le déficit maximal est relevé à Mont-de-Marsan (Landes) avec -6,7 jours et l'excédent maximal a été observé au cap Pertusato (Corse) avec +3 jours. d'insolation mensuelle ont été battus : Brétigny-sur-Orge (Essonne) : 170 h 55 (ancien record : $144 \mathrm{~h}$ en 1995) avec 7 jours d'insolation diurne continue. Alençon (Orne) : $172 \mathrm{~h}$ (ancien record : $170 \mathrm{~h}$ en 1947). pointes dépassant souvent les $100 \mathrm{~km} / \mathrm{h}$ près des côtes de Manche, comme au Capde-La-Hève les 7, 8 et 9. Le vent de sudouest souffle jusqu'à $104 \mathrm{~km} / \mathrm{h}$ à l'Île-deBatz le 8 ; il atteint son maximum $137 \mathrm{~km} / \mathrm{h}$ au cap de La Hague. Sur les 
hauteurs de l'Auvergne, il se fait également ressentir (100 et $104 \mathrm{~km} / \mathrm{h}$ à Orcines à 1415 mètres d'altitude les 7 et 9).

Les 10 et 11, le flux s'oriente au nord-ouest ; les rafales atteignent $118 \mathrm{~km} / \mathrm{h}$ le 11 à la pointe du Raz, $115 \mathrm{~km} / \mathrm{h}$ à la Pointe-duGrouin. Le vent de nord-ouest se renforce également sur les hauteurs : $140 \mathrm{~km} / \mathrm{h}$ à Orcines le 10,130 et $155 \mathrm{~km} / \mathrm{h}$ à Chamonix-Mont-Blanc (3 845 mètres) les 10 et $11,126 \mathrm{~km} / \mathrm{h}$ au mont Aigoual le 11 . Persistance d'un fort courant de nord-ouest du 12 au 15 : durant cette période, le vent de nord-ouest associé à de l'air froid souffle sur une bonne partie du pays. Le 12 , les rafales atteignent $104 \mathrm{~km} / \mathrm{h}$ à Millau, $122 \mathrm{~km} / \mathrm{h}$ à Leucate, $115 \mathrm{~km} / \mathrm{h}$ à Mandelieu-La Napoule, $104 \mathrm{~km} / \mathrm{h}$ à Bastia, $115 \mathrm{~km} / \mathrm{h}$ à Soumont, $118 \mathrm{~km} / \mathrm{h}$ à HyèresLe Levant, $130 \mathrm{~km} / \mathrm{h}$ à Orcines. En
Bretagne et en Normandie, on relève 137 $\mathrm{km} / \mathrm{h}$ à Brignogan et à la pointe du Raz et $108 \mathrm{~km} / \mathrm{h}$ à Carteret. À Leucate, on observe $104 \mathrm{~km} / \mathrm{h}$ les 13 et 14 . Les rafales atteignent $140 \mathrm{~km} / \mathrm{h}$ le 14 au Cap-Béar.

Le 17 et surtout le 18, c'est au tour du vent de sud de se faire sentir. À Dourgne dans le Tarn, le vent d'autan frôle les $110 \mathrm{~km} / \mathrm{h}$ dans la journée du 18 .

Les 28 et 29, le vent d'est est fort sur la côte orientale de la Corse. Il atteint les $104 \mathrm{~km} / \mathrm{h}$ en rafales à Figari.

Le nombre de jours avec vent fort atteint 20 à la pointe du Raz (Finistère). Fortement déficitaire sur la pointe bretonne et plus légèrement sur la majeure partie de l'Hexagone, il est excédentaire dans le Sud-Est, sur le Cotentin, dans les Pays de la Loire, dans le Nord-Est et sur la moitié nord de la Corse.

\section{Écart à la normale de la température moyenne (degrés Celsius)}

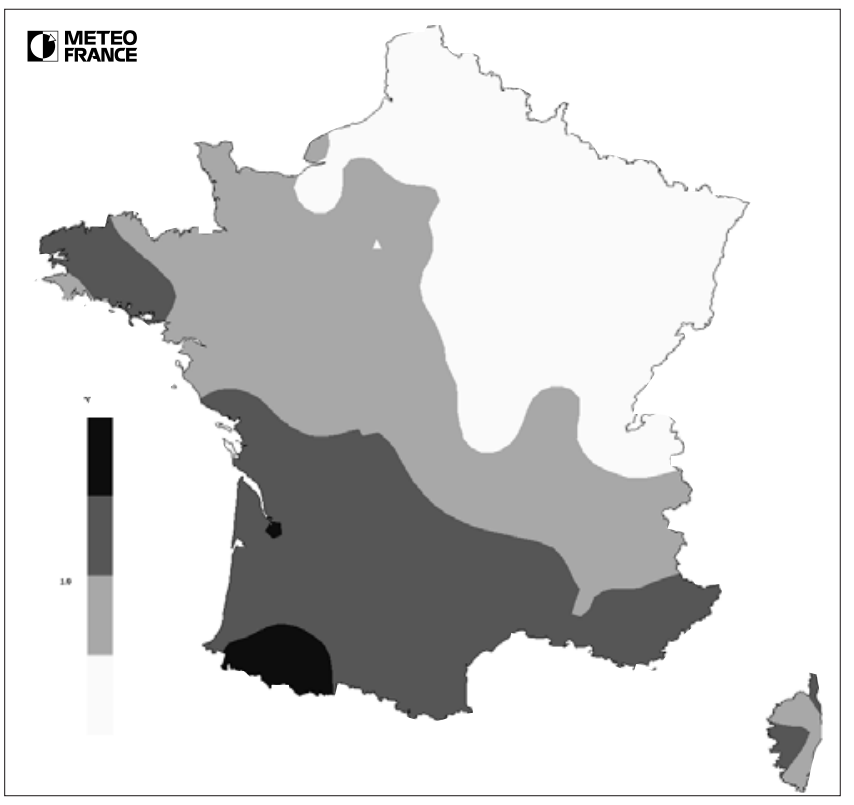

\section{Rapport à la normale des hauteurs de précipitations (pour cent)}

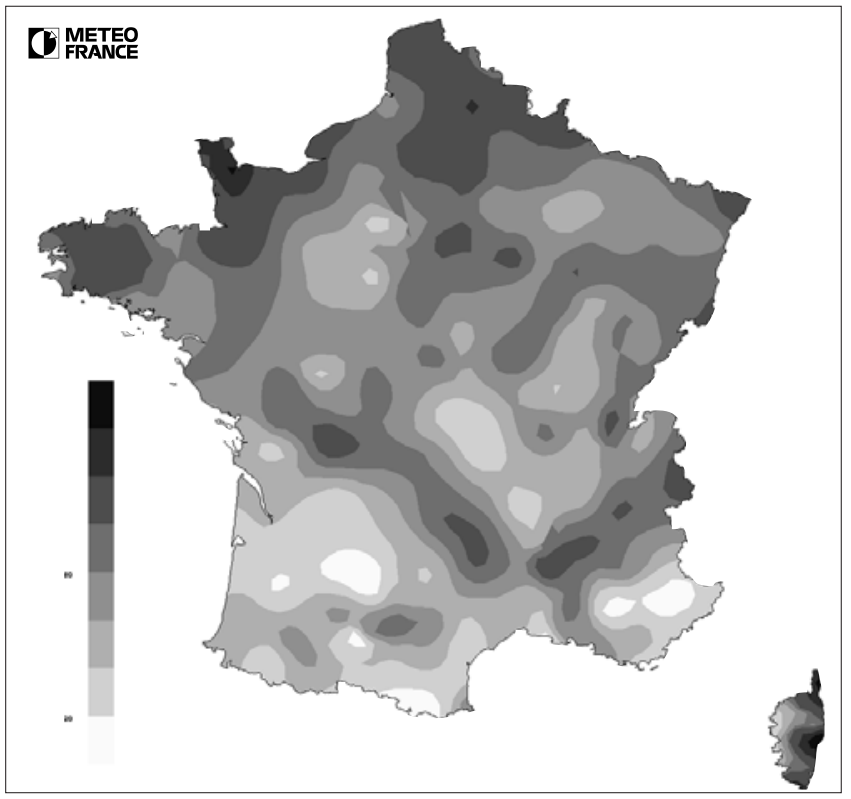

